If only because cardiotocography has been shown to reduce the rate of neonatal convulsions after complicated labours its use should not be abandoned. Maternity units should have clear, explicit guidelines on the grounds for recommending cardiotocography so that the clinical and legal responsibility does not lie with a junior obstetrician or midwife. Suitable grounds might include prolonged, augmented, or induced labours; multiple pregnancies; thick meconium staining of amniotic fluid; and a growth retarded or preterm fetus. Audible heart rate decelerations also indicate a need for electronic monitoring.

Improvements in the results of monitoring will come not only by better identification and interpretation of abnormalities of the fetal heart rate but also through better responses by attendants to unusual patterns. Simple measures (stopping the oxytocin infusion, altering the mother's posture, restoring blood pressure to normal) often suffice; techniques such as amnioinfusion deserve further investigation as alternatives to operative delivery. ${ }^{13}$

Recording the fetal heart rate has become a fixed feature of intrapartum care; we need to reconsider and remember that it is an extremely limited method for assessing the health of the fetus. We do not expect doctors to run an intensive care unit simply by measuring the pulse rates of their patients. The status of intrapartum fetal heart rate recording (whether assessed by ear or by machine) is similar to that of the neonatal test frequently used to assess the effectiveness of the measure-the much maligned but universally used Apgar score. Both are clearly unsatisfactory, but a practical and popular alternative has not yet emerged for either. The search for better techniques of fetal assessment during labour continues. For "high risk" fetuses, waveform analysis of the electrocardiogram shows promise ${ }^{6}$; oximetry still has some way to go. ${ }^{14}$

JAMES P NEILSON Professor of Obstetrics and Gynaecology

Royal Liverpool University Hospital,

Liverpool L7 8XP
1 Janbu T, Nesheim B-I. Uterine artery blood velocities during contractions in pregnancy and labour related to intrauterine pressure. Br $\mathcal{O}$ Obstet Gynaecol 1987;94:1150-5.
2 Chalmers I, Dickersin K, Chalmers TC. Getting to grips with Archie Cochrane's agenda. BMF 1992;305:786-8.
3 Grant A. Monitoring the fetus during labour. In: Chalmers I, Enkin M, Keirse MJNC, eds. Effective care in pregnancy and childbirh. Oxford: Oxford University Press, 1989:846-82.
4 Grant AM. EFM + scalp sampling vs intermittent auscultation in labour. In: Chalmers I, ed. Oxford database of perinatal trials. Version 1.3, disk issue 8. Oxford: Oxford University Press, 1992:record 3297.
5 Grant AM. EFM alone vs intermittent auscultation in labour. In: Chalmers I, ed. Oxford database of perinatal trials. Version 1.3, disk issue 8. Oxford: Oxford University Press, 1992:record 3298.
6 Westgate J, Harris M, Curnow JSH, Greene KR. Randomised trial of cardiotocography alone or with ST waveform analysis for intrapartum monitoring. Lancet 1992;340:194-8.
7 MacDonald D, Grant AM, Sheridan-Pereira M, Boylan P, Chalmers I. The Dublin randomized collo
Grant AM, O'Brien N, Joy M-T, Hennessy E, MacDonald D. Cerebral palsy among children born during the Dublin randomised trial of intrapartum monitoring. Lancet 1989;ii:1233-6.
9 Grant AM. EFM vs intermittent auscultation in labour. In: Chalmers I, ed. Oxford database of perinatal trials. Version 1.3, disk issue 8. Oxford: Oxford University Press, 1992:record 3884.
10 Garcia J, Corry M, MacDonald D, Elbourne D, Grant A. Mothers' views of continuous electronic fetal heart rate monitoring and intermittent auscultation in a randomized controlled trial. Birth 1985;12:79-85.
11 Ingemarsson I, Arulkumaran S, Ingemarsson E, Tamby Raja RL, Ratnam SS. Admission test-a screening test for fetal distress in labor. Obstet Gynecol 1986;68:800-6.
12 Cockburn JE, Pearce JM, Chamberlain GVPC. Intrapartum fetal monitoring. Lancet 1992;340:610.
13 Hofmeyr GJ. Amnioinfusion: a question of benefits and risks. Brf Obstet Gynaecol 1992;99:449-51.
14 Johnson N, Johnson VA, Fisher J, Jobbings B, Bannister J, Lilford RJ. Fetal monitoring with pulse oximetry. Br f Obstet Gynaecol 1991;98:36-41.

\title{
Mothering skills of women with mental illness
}

\author{
Not enough known about the postpartum period
}

The traditional role of mother and baby units in psychiatry has been to treat acute postpartum mental illness without separating the mother from the infant. Increasingly, however, such units are being asked to assess the quality of mothering by women with chronic disorders, particularly schizophrenia but also poor control of impulses. Many referrals are initiated by social services departments, possibly as a result of growing pressure to protect children in accordance with their responsibility under the Children Act. As a result, psychiatrists take on the crucial rule of assessor and expert witness in child care cases in which the mother has a mental or behavioural disorder. How should specialist services respond to this challenge? What is known about the capacity of mentally ill mothers to care for their babies?

Maternal mental illness may adversely affect a child both directly - causing neglect, physical harm, and psychological upset-and indirectly - through associated features of the illness such as marital disharmony and repeated admissions to hospital. ${ }^{1}$ In a series of women who had killed their children $84 \%$ had mental and behavioural disorders, but diagnoses varied, with $16 \%$ being psychotic and personality disorder being the commonest diagnosis. ${ }^{2}$ Acute schizophrenia can severely impair mothering skills when maternal distress leads to distraction and neglect. Close supervision of patients is needed when they have delusions or hallucinations concerning the baby. Impairment of warmth and rigid or anxious mothering styles also occur during the acute illness, and improvement may be delayed for up to one year, depending on the premorbid social adjustment. ${ }^{3}$ In women with chronic schizophrenia blunted or incongruous emotions, poor moti- vation, disturbed behaviour, and lack of responsiveness to the child's cues may all affect mother-infant interaction. Maternal responsiveness is thought to be important in cognitive development $^{4}$ and for secure mother-infant attachment ${ }^{5}$ : thus infants of mothers with chronic schizophrenia are at risk of disrupted bonding and its sequelae of impaired social behaviour and problem solving. ${ }^{6.8}$

Personality disorder is the parental diagnosis most associated with emotional and behavioural disorders in children, particularly when they have been exposed to hostile behaviour.' In addition, lack of warmth and chaotic behaviour may result in child neglect, and impulsiveness and reduced tolerance to stress can be expected to increase the risk of physical abuse. Abuse and neglect are also commonly seen in children of mothers with learning disabilities and are particularly common if the fathers also suffer from learning disabilities or if the children themselves are of normal IQ. ${ }^{9}$

In severe depression apathy can lead to neglect, irritability to physical harm, and depressive delusions to infanticide. Cognitive impairment in children may follow milder postpartum depression and may be detectable four years after the resolution of maternal symptoms. ${ }^{10}$ Behavioural problems at 3-4 years of age have also been associated with maternal depression, both concurrent ${ }^{11}$ and postnatal. ${ }^{12}$ In the longer term the offspring of depressed mothers are more likely to suffer from childhood depression. ${ }^{1}$

Despite these findings key questions remain concerning the prediction of successful mothering after postpartum psychiatric disorder, and there is little evidence on how maternal 
competence can best be assessed. One descriptive account of assessment recommends full psychiatric evaluation, comprehensive observations of the mothers with their infants, and interviews with relatives to estimate future support. ${ }^{13}$ Although this commonsense approach is probably used in most mother and baby units, including those that now admit mothers with chronic illness for preliminary assessment and subsequent training in mothering skills, the practice is open to criticism. To be clinically useful, observations must be simple enough to be made by multiple carers on a psychiatric ward; excessive simplicity, however, may undermine their value. Also in doubt is their predictive validity, as no study has determined mother-infant interaction after discharge from a mother and baby unit or identified which observations during the admission are most crucial to prognosis.

A potential problem now exists in deciding who should pay for such detailed assessments: mother and baby units must attract referrals from outside their district to remain financially viable, and their high ratio of staff to patients is expensive. Health authorities will be asked to purchase this form of care, and social services will be required to pay directly for the advice they need. If they do not decisions may be made without the benefit of comprehensive observations and specialist experience. Some children may be removed prematurely from their families while others may be entrusted to incapable mothers whose difficulties remain undetected and untreated.

LOUIS APPLEBY Senior Lecturer CHRIS DICKENS Registrar

Department of Psychiatry,

Withington Hospital,

Manchester M20 8LR

1 Rutter M, Quinton D. Parental psychiatric disorder: effects on children. Psychol Med 1984;14:853

2 d'Orban PT. Women who kill their children. Br F Psychiatry 1979;134:560-71.

Rutter M. Family and school influences on cognitive development. $\mathcal{f}$ Child Psychol Psychiatr 1985;26:683-704.

Rodnick EH, Goldstein MJ. Premorbid adjustment and the recovery of mothering function in acute schizophrenic women. $f$ Abnorm Psychol 1974;83:623-8.

5 Blehar MC, Lieberman AF, Ainsworth MDS. Early face-to-face interaction and its relation to late infant-mother interaction. Child Dev 1977;48:182-94.

6 Pastor DL. The quality of mother-infant attachment and its relation to toddlers' initial sociability with peers. Developmental Psychology 1981;17:326-335.

Matas L, Arend RA, Sroufe LA. Continuity of adaption in the second year: the relationship between quality of attachment and later competence. Child Dev 1978;49:547-56. between quality of attachment and later competence. Child Dev 1978;49:547-56.

9 Accardo PJ, Whitman BY. Children of mentally retarded parents. Am f Dis Child 1990;144: $69-70$.

10 Cogill K, Caplan HL, Alexandra H, Robson KM, Kumar R. Impact of maternal postnatal depression on cognitive development of young children. $B M Y$ 1986;292:1165-7.

1 Caplan HK, Cogill SR, Alexandra H, Robson KM, Katz R, Kumar R. Maternal depression and the emotional development of the child. Br f Psychiatry 1989;154:818-22.

12 Wrate RM, Rooney AC, Thomas PF, Cox JL. Postnatal depression and child development. A three year follow-up study. Br f Psychiatry 1985;146:622-7.

13 Stewart D, Gangbar R. Psychiatric assessment of competency to care for the newborn. Can $f$ Psychiatry 1984;29:583-9.

\section{Screening newborn infants for Duchenne muscular dystrophy}

\section{Acceptable to parents}

Bradley and colleagues describe a programme for screening newborn infants for Duchenne muscular dystrophy on $\mathrm{p} 357 .{ }^{1}$ Symptoms of this severe $\mathrm{X}$ linked inherited disorder usually occur before the age of 6 , and the patient is bedridden by 12 and often dead by $20 .^{2}$

Newborn screening for genetic and other disorders is usually confined to diseases for which there is treatmenteither a change of diet (as in phenylketonuria and galactosaemia) or replacement therapy (as in hypothyroidism) - or in which there is a chance of affecting morbidity or mortality early in life, as occurs in the haemoglobinopathies (particularly sickle cell disease). Screening for disorders in which the clinical course cannot be altered is generally avoided: a programme of newborn screening for disorders like Duchenne muscular dystrophy could therefore create an ethical nightmare. Bradley and colleagues are well aware of these problems as they seek to justify newborn screening for this untreatable genetic disorder.

The authors argue that newborn screening for Duchenne muscular dystrophy decreases emotional distress because it avoids delays in diagnosing the disease, and without screening two or more boys could be born with the condition before parents realised that they were at risk of having an affected child. Accordingly, if an infant with Duchenne muscular dystrophy is identified early the family has the option of prenatal diagnosis for future pregnancies. On the other hand, neonatal diagnosis could interfere with normal emotional growth and the child's interaction with his family.

Data are presented from a programme throughout Wales, which began in July 1990 with several aims. These included giving families reproductive choice in future pregnancies, enabling them to plan for the future with a disabled child, avoiding the experience of a delayed diagnosis, and identifying a presymptomatic cohort who might benefit from future treatments. Parents were given the option of having their male infants screened for Duchenne muscular dystrophy in addition to the routine tests for phenylketonuria and congenital hypothyroidism. Informed consent was sought from parents, who were given an information sheet.

Blood was obtained by heel prick. Out of 16 cases that tested positive for creatine kinase, nine were confirmed, giving an incidence of 1:3082 births. Seven infants had a transient increase in creatine kinase activity; the authors say that no evidence exists that such rises are associated with the development of muscular dystrophy later in life. The refusal rate has varied between $5 \%$ and $6 \%$.

Considering the possibility of emotional trauma resulting from such a programme is crucial. Interestingly, only one of the nine families with an affected child had an above average level of emotional trauma. The other parents wanted to know about their son's condition early in life. Undoubtedly, the low incidence of emotional trauma was due in part to the careful protocol, which acknowledged that families risked finding out that their apparently healthy baby had a lethal disorder. Accordingly, the programme was designed so that it could be modified in the light of experience, and families could choose to discontinue investigation at every stage. Readers should pay particular attention to the careful, extensive community outreach organisation, which undoubtedly led to the minimal emotional impact of the programme.

University of Chicago,

JAMES E BOWMAN Professor of Pathology

Chicago, Illinois 60637, USA

\footnotetext{
Bradley DM, Parsons PE, Clarke AJ. Experience with screening newborns for Duchenne muscular dystrophy in Wales. $B M \mathcal{F}$ 1993:306:357-60.

McKusick VA. Mendelian inheritance in man: catalogs of autosomal dominant, autosomal recessive, and $X$-linked phenotypes. 10th ed. Baltimore: Johns Hopkins University Press, 1992:1916-26.
} 